\title{
The Dynamic Insulin
}

Sensitivity and Secretion Test (DISST)

\section{A novel method of insulin sensitivity}

For the definitive version of this article, see:

McAuley, K.A., Berkeley, J.E., Docherty, P.D., Lotz, T.F., Te Morenaga, L.A., Shaw, G.M., Williams, S.M., Chase, J.G. and Mann, J.I. (2011) The Dynamic Insulin Sensitivity and Secretion Test - a novel measure of insulin sensitivity. Metabolism, 60(12), 1748-1756.

http://dx.doi.org/10.1016/j. metabol.2011.05.009. 
2 The Dynamic Insulin Sensitivity and Secretion Test (DISST) - a novel

3 measure of insulin sensitivity.

4 Kirsten A. McAuley ${ }^{1}$, Juliet E. Berkeley², Paul D. Docherty ${ }^{3}$, Thomas F. Lotz ${ }^{3}$, Lisa A.

5 Te Morenga ${ }^{4,5}$, Geoff M. Shaw ${ }^{2}$, Sheila M. Williams ${ }^{6}$, J. Geoffrey Chase ${ }^{3}$, Jim I. Mann ${ }^{1,4}$.

6

$7 \quad{ }^{1}$ Edgar National Centre for Diabetes and Obesity Research, University of Otago, Dunedin,

8 New Zealand.

$9 \quad{ }^{2}$ Christchurch School of Medicine, University of Otago, Christchurch, New Zealand.

$10{ }^{3}$ Department of Mechanical Engineering, University of Canterbury, Christchurch, New

11 Zealand.

$12 \quad{ }^{4}$ Department of Human Nutrition, University of Otago, Dunedin, New Zealand.

$13{ }^{5}$ Riddet Institute, Palmerton North, New Zealand

$14{ }^{6}$ Department of Preventive and Social Medicine, University of Otago, Dunedin, New Zealand

15 Conflict of interest statement: The authors declare no conflict of interest.

16 Corresponding author: Dr Kirsten McAuley, Edgar National Centre for Diabetes and

17 Obesity Research, Department of Medicine, PO Box 913, Dunedin 9054, New Zealand.

18 Phone: +6434798153 and/or +6421893831

19 Fax: +6434797958 (Attention: C/- Victoria Farmer)

20 Email: kirsten.mcauley@otago.ac.nz

21 Word count of abstract: 249

22 Word count of text: 2818

23 Number of tables: 4

24 Number of figures: 4

25 Number of references: 32

26 Running title: New Short Test to Measure Insulin Sensitivity 


\section{Abstract}

Objective: To validate the methodology for the Dynamic Insulin Sensitivity and Secretion Test (DISST) and to demonstrate its potential in clinical and research settings.

Methods: 123 men and women had routine clinical and biochemical measurements, an oral glucose tolerance test and a DISST. For the DISST, participants were cannulated for blood sampling and bolus administration. Blood samples were drawn at $t=0,10,15,25$ and 35 minutes for measurement of glucose, insulin and C-peptide. A 10g bolus of intravenous glucose at $t=5$ minutes and $1 \mathrm{U}$ of intravenous insulin immediately after the $t=15$ minute sample were given. Fifty participants also had a hyperinsulinaemic euglycaemic clamp. Relationships between DISST insulin sensitivity (SI) and the clamp, and both DISST SI and secretion and other metabolic variables were measured.

Results: A Bland-Altman plot showed little bias in the comparison of DISST with the clamp; with DISST underestimating the glucose clamp by $0.1 \cdot 10^{-2} \cdot \mathrm{mg} \cdot 1 \cdot \mathrm{kg}^{-1} \cdot \mathrm{min}^{-1} \cdot \mathrm{pmol}^{-1}(90 \% \mathrm{CI}$ 0.2 to 0 ). The correlation between $S I$ as measured by DISST and the clamp was 0.82 , the c unit for the ROC analysis for the two tests was 0.96. Metabolic variables showed significant correlations with DISST IS, and the second phase of insulin release. DISST also appears able to distinguish different insulin secretion patterns in individuals with identical SI values.

Conclusions: DISST is a simple, dynamic test that compares favourably with the clamp in assessing SI and allows simultaneous assessment of insulin secretion. DISST has the potential to provide even more information about the pathophysiology of diabetes than more complicated tests.

Key Words: insulin sensitivity, beta cell function, insulin secretion, insulin resistance, type 2 diabetes mellitus.

Abbreviations: BMI: body mass index. DBP: Diastolic blood pressure. DISST: dynamic insulin sensitivity and secretion test. FPG: fasting plasma glucose. HOMA: homeostasis model assessment. IGT: impaired glucose tolerance. NGT: normo-glucose tolerance. IQR: interquartile range. IVGTT: intravenous glucose tolerance test. NGT: normal glucose tolerance. OGTT: oral glucose tolerance test. ROC: receiver operator curve. SBP: systolic blood pressure. SI: insulin sensitivity. 


\section{Introduction}

Insulin resistance and $\beta$-cell dysfunction are prerequisites for the development of impaired fasting glucose, impaired glucose tolerance (IGT) and type 2 diabetes mellitus. However, the lack of a relatively simple test to reliably quantify both insulin sensitivity and secretion make it difficult to examine heterogeneity in epidemiological studies of prediabetes and diabetes and explore pathophysiology in studies of prevention and treatment. We have described a simple test - DISST [1, 2] which can provide quantitative measures of insulin sensitivity and insulin secretion.

The present paper has utilised a simple version of the DISST which involves five blood samples taken over a 35-minute protocol that uses low-dose, intravenous glucose (10g) and insulin (1U) boluses as stimulus. Thus, it is relatively short, and considerably less labour intensive than the gold-standard glucose clamp. The DISST model and identification method enable the sparse sampling protocol by fitting and refining physiological responses to the measured data [3, 4]. Unlike previous models, the DISST model of glucose and insulin kinetics accounts for patient-specific losses of insulin to the liver and the kidneys, saturation of insulin clearance at high concentrations and diffusion and mass conservation of insulin between the plasma and the interstitium [4]. In addition to assessing insulin sensitivity the test can be used to assess ß-cell function using established methods [5]. This aspect of the DISST is not novel.

The availability of such a test which can physiologically assess insulin sensitivity and simultaneously estimate insulin secretion provides the potential to explore heterogeneity in those who are currently labelled with the diagnosis of metabolic syndrome, prediabetes or type 2 diabetes mellitus, and to further understand responses to treatment with lifestyle measures and pharmacology.

This paper provides a validation of the DISST in the assessment of insulin sensitivity and illustrates its potential use.

\section{Methods}

Data from two separate studies undertaken by the same group of investigators have been combined. The first study cohort included 10 lean $(\mathrm{BMI}<25), 20$ overweight $(\mathrm{BMI}>25,<30)$ 
and 20 obese (BMI>30) participants, with even gender distribution in each category. The second study cohort included 73 women who were considered at-risk of metabolic diseases either by a BMI $>25$ or BMI $>23$ and a family history of diabetes. Participants were excluded if they suffered from any major medical or psychiatric illness or were known to have diabetes. Ethical approval for the first study was from the Upper South A Regional Ethics Committee. The second study was approved by the University of Otago Ethics Committee.

All 123 participants had weight, waist circumference (the midpoint of the lowest rib and highest part of the hip) and resting blood pressure measured. The 50 participants in the first study underwent a glucose clamp, the 4-sample OGTT and DISST protocols within 8 days, with at least one day between tests. The tests were given in random order such that each of the six possible combinations were equally represented. A pre-randomised test order was allocated to each participant based on order of recruitment. Participants of the second study underwent the DISST and the 2-sample OGTT in order to classify them as having a normal, or impaired glucose tolerance or type 2 diabetes [6]. All participants fasted from 10pm the night before each test and the tests were begun at 9am.

\section{OGTT protocol}

Fifty participants from the first study had an OGTT for assessment of insulin sensitivity using the Matsuda method [7]. Participants were given a standard 75g oral glucose load after a fasting blood sample. Further blood samples were collected at 30, 60 and 120 minutes. HOMA was also calculated for the first study participants using the basal assays of the OGTT and previously published methods [8, 9].

\section{DISST protocol}

Participants had a cannula inserted into the antecubital fossa for blood sampling and bolus administration. Blood samples were drawn at $t=0,10,15,25$ and 35 minutes and glucose, insulin and C-peptide was measured on these samples. A $10 \mathrm{~g}$ bolus of intravenous glucose was given at $t=5$ minutes and $1 \mathrm{U}$ of Actrapid insulin was given immediately after the $t=15$ minute sample. Participants were required to remain at the clinic for 30 minutes after the test and were provided with a small meal or snack.

The parameter identification methods of dynamic tests (such as the DISST) are sensitive to the timing of samples. Thus, the actual sample times were recorded. The integral method is 
used to identify model-based insulin sensitivity $(S I)$, glucose distribution volume ( $\mathrm{Vg})$, firstpass $\left(x_{L}\right)$ and subsequent hepatic insulin clearance $\left(n_{L}\right)[3,10]$. Metrics of $\beta$ cell function are derived from insulin production profiles that are deconvolved from interpolated C-peptide data following the established method of Van Cauter et al. [3, 5]. The DISST model and identification method are briefly repeated in Appendix 1.

Three metrics were used to quantify $\beta$ cell function. The basal rate $\left(U_{b}\right)$ indicates the rate of insulin production the participant requires to maintain a constant fasting glucose measurement. $\mathrm{AUC}_{10}$ measures the first phase insulin production and is defined as the amount of insulin produced above the basal rate during the ten minutes after the glucose bolus. $\mathrm{AUC}_{2 n d}$ quantifies the participant's second phase of insulin production as the total amount of insulin produced during the 20 minutes after the period measured by $\mathrm{AUC}_{10}$.

The DISST method used in this study is a simpler version of the original DISST [3, 4] using 5 blood samples instead of 9. The impact of such sparse sampling on insulin sensitivity and insulin secretion metrics have been shown to be limited in previous studies [4, 11, 12]. Previous analysis by Docherty et al. found that insulin sensitivity and production values were barely affected by the omission of samples from the frequently sampled protocol used in the DISST pilot study [12]. The five-sample method was not significantly different from the original 9-sample method. The correlations between the outcomes of the pilot sampling protocol and the sampling protocol used here were $\mathrm{r}=0.90,1.0,1.0$, and 0.89 for $S I, U_{b}$, $\mathrm{AUC}_{10}$, and $\mathrm{AUC}_{2 \mathrm{nd}}$, respectively.

\section{Glucose Clamp protocol}

The 50 participants in the first study underwent a glucose clamp. Participants had two cannulae inserted: one in the antecubital fossa; the other, a retrograde cannula, inserted in the dorsum of the hand. The hand was heated so that arterialised blood was obtained for sampling. Insulin was infused at $280 \mathrm{pmol} / \mathrm{min} / \mathrm{m}^{2}$ and glucose was infused to achieve a target glucose concentration of $81 \mathrm{mg} / \mathrm{dl}$, or at the fasting level if this was between 72 and $90 \mathrm{mg} / \mathrm{dl}$. The test lasted for 2 to 2.5 hours and data from the last 40 minutes was used to calculate insulin sensitivity index (ISI) in $\mathrm{mg} \cdot \mathrm{l} \cdot \mathrm{kg}^{-1} \cdot \mathrm{min}^{-1} \cdot \mathrm{pmol}^{-1}$ [13]. Participants were required to remain at the clinic for 30 minutes after the test and were provided with a small meal or snack. 
As the standard DISST and the clamp SI values have different units, a conversion needs to be made to compare the magnitude of values across the two different tests. The model-based SI identified by the DISST measures the glucose disposal as a function of the available glucose, glucose distribution volume and the modelled interstitial insulin. However, the clamp measures as a function of the absolute glucose disposal, steady-state plasma insulin concentration and the participant's bodyweight. Thus to achieve common units the DISST SI values must be converted:

$$
I S I_{\text {DISST }}=S I_{\text {modelled }} \frac{1000 \cdot \mathrm{Gb} \cdot \mathrm{Vg} \cdot \gamma}{B W}
$$

where: $G_{b}$ is the basal glucose concentration $\left[\mathrm{mmol} \cdot \mathrm{l}^{-1}\right], \mathrm{Vg}$ is the identified distribution volume of glucose [1], $B W$ is bodyweight [kg], $\gamma$ is the steady state ratio between plasma and interstitial insulin (0.5) [14], and the '1000' coefficient converts from dl to l and multiplies by 100 in accordance with the standard practice for reporting clamp metrics.

\section{Laboratory analysis}

Glucose values for the first study were analysed using YSI 2300 stat plus Glucose and LLactate analyser using whole blood. These were converted to plasma glucose with the equation recommended by the analyser manufacturer:

$$
G_{\text {plasma }}=\frac{G_{\text {wholebloodglucose }}}{1-\left(2.4 \cdot 10^{-3} \cdot \text { Haematocrit }(\%)\right)}
$$

Plasma glucose levels taken in the second study were measured enzymatically with Roche kits and calibrators on a Cobas Mira Analyser. Samples for insulin and C-peptide were separated immediately and frozen. Measurements of insulin were undertaken by the Endolab, Canterbury Health Laboratories for the first study and by the University of Otago Nutrition Laboratory for the second study. Both laboratories used Roche Elecsys ${ }^{\circledR}$ after Peg precipitation of immunoglobulins (Roche Diagnostics, Mannheim, Germany). Consistency between laboratories was maintained. All C-peptide measurements were undertaken by Endolab, Canterbury Health Laboratories using the Roche Elecsys ${ }^{\circledR}$ method. Serum cholesterol and triglycerides were measured enzymatically with Roche kits and HDL was measured in the supernatant after precipitation of apolipoprotein B containing lipoproteins with phosphotungstate/magnesium chloride solution [15].

\section{Statistical methods}


The data are presented as means and standard deviations or median and upper and lower quartiles. Correlations were used to describe the associations between the insulin sensitivity values. A Bland Altman plot was used to compare the DISST with the glucose clamp. Analysis of variance was used to compare the three groups derived from the first insulin phase $\left(\mathrm{AUC}_{10}\right)$ and those derived from the second insulin phase $\left(\mathrm{AUC}_{2 \mathrm{nd}}\right)$. Comparisons between those with IGT and those with NGT are also presented.

\section{Results}

The range of DISST insulin sensitivity values for the 123 individuals was 0.2 to $3.4 \cdot 10^{-4}$ $\cdot l \cdot \mathrm{pmol}^{-1} \cdot \mathrm{min}^{-1}$ with a mean of 1.1 (SD 0.64 ), median 1.0 (IQR 0.7 to 1.4 ). The range for insulin sensitivity estimated by the glucose clamp $(\mathrm{n}=50)$ was 0.1 to $2.3 \cdot 10^{-2} \cdot \mathrm{mg} \cdot \mathrm{l} \cdot \mathrm{kg}^{-1} \cdot \mathrm{min}^{-}$ ${ }^{1} \cdot \mathrm{pmol}^{-1}$, mean 1.0 (SD 0.61), median 0.9 (IQR 0.6 to 1.4 ).

The Bland-Altman plot (Figure 1) shows the bias between the two tests, where the DISST underestimated the glucose clamp by $0.1 \cdot 10^{-2} \cdot \mathrm{mg} \cdot 1 \cdot \mathrm{kg}^{-1} \cdot \mathrm{min}^{-1} \cdot \mathrm{pmol}^{-1}$ (95\% CI -0.2 to 0.0). The limits of agreement were -0.9 to $0.7 \cdot 10^{-2} \cdot \mathrm{mg} \cdot 1 \cdot \mathrm{kg}^{-1} \cdot \mathrm{min}^{-1} \cdot \mathrm{pmol}^{-1}$. Figure 2 shows the correlation between the DISST and the glucose clamp ( $r=0.82)$. Figure 3 presents a ROC curve for the DISST compared to the glucose clamp (c unit=0.96 using an insulin resistance cut off for the glucose clamp of $\left.1.0 \cdot 10^{-2} \cdot \mathrm{mg} \cdot 1 \cdot \mathrm{kg}^{-1} \cdot \mathrm{min}^{-1} \cdot \mathrm{pmol}^{-1}[9]\right)$.

Correlations between the DISST and the variables known to be associated with insulin resistance are shown in Table 1 as well as the correlations between the DISST and the HOMA and the Matsuda index.

Characteristics of those separated into tertiles of first phase and second phase insulin secretion are shown in Tables 2 and 3. Of note, those with IGT were spread evenly across the tertiles of first phase insulin secretion. However, second phase insulin secretion was significantly associated with all of the features of the metabolic syndrome. Table 4 compares insulin secretion metrics across the NGT and IGT subgroups. In accordance with previous observations [16-18], the second phase insulin secretion was significantly higher in those with IGT.

Figure 4 shows the results of the DISST test for insulin sensitivity and insulin secretion metrics for four insulin resistant participants. All of the examples in this figure had the same 
230 insulin sensitivity measured by the clamp $\left(0.8 \cdot 10^{-2} \cdot \mathrm{mg} \cdot 1 \cdot \mathrm{kg}^{-1} \cdot \mathrm{min}^{-1} \cdot \mathrm{pmol}^{-1}\right)$, however the

231 DISST profiles showed clear differences between these individuals. The range of insulin 232 sensitivity estimated by the DISST was 0.95 to $1.36 \cdot 10^{-4} \cdot 1 \cdot \mathrm{pmol}^{-1} \cdot \mathrm{min}^{-1}$ for these participants.

233 However, of particular note were the distinct insulin production characteristics of these 234 participants. Participants A and B showed contrasting profiles to participants C and D in 235 terms of the magnitude of first phase release of insulin. Participant $C$ had an increased second 236 phase, and blunted first phase of insulin production, which coupled with an inability to return 237 to the basal glucose concentration within 30 minutes, indicated insulin resistance and $\beta$ cell 238 dysfunction for this participant.

240 No serious adverse events were observed in participants and there were no episodes of 241 symptomatic hypoglycaemia following the DISST.

\section{Discussion}

244 The DISST estimates both insulin sensitivity and $\beta$ cell function including the first and second phases of insulin release. The protocol is well accepted by participants, and is straightforward to perform. The low-dose and low-intensity DISST protocol is unique in that it results in insulin concentrations that are comparable with daily excursions and are not affected by dosedependent saturation effects [19] whereas established tests rely on non-physiological doses that exceed saturation level for insulin action [20, 21]. Thus, the model-based DISST can more directly account for dosing differences than the simpler M/I model of glucose clearance and uptake used in the clamp assessment, which varies directly with the insulin dosing due to insulin effect saturation [20,21]. As a result, DISST SI values are more directly comparable across studies [3].

The DISST concurrently allows an assessment of insulin secretion with insulin sensitivity. The insulin secretion identification method was validated by Van Cauter et al. [5] and has been used by many leading insulin sensitivity research groups [22-25]. The second phase values of insulin secretion obtained from the low-intensity DISST correlated well with metabolic risk factors, and distinguished IGT and NGT subgroups. The DISST offers the possibility of relating the insulin secretion rate to their insulin sensitivity status, which is potentially useful in research and clinical practice. Insulin secretion typically increases with insulin resistance in the early stages of IGT and type 2 diabetes, but declines as $\beta$ cell function 263 is lost [13, 26, 27]. Thus, as illustrated in Figure 4, apparently healthy NGT individuals can 
have insulin production rates similar to those of individuals that have considerable loss of $\beta$ cell function. Current tests do not distinguish between these individuals with different insulin secretion responses [16].

The DISST insulin sensitivity values were converted to mimic the units of the gold-standard clamp, and thus could be compared to clamp values in terms of both correlation and bias. Furthermore, Table 1 compared the DISST insulin sensitivity values to co-factors related to insulin resistance and produced expected outcomes [28]. The moderate correlations presented in Table 1 must be considered with respect to the low resolution of the co-factors presented in terms of characterising insulin resistance.

In contrast to insulin sensitivity, there is no established gold standard for the evaluation of insulin secretion. Thus, this investigation has evaluated the insulin secretion values estimated by the DISST by comparison with established metabolic markers of insulin resistance. Furthermore, the low-intensity 5 sample protocol has been validated by Lotz et al. [11].

The DISST requires a significantly less intensive protocol than insulin sensitivity tests that produce similar correlations to the clamp [29-31]. The DISST can achieve this level of accuracy with improved parameter identification methods [10] and the adoption of a single model variable for glucose decay. The identification of two metrics that model glucose clearance has been an issue in previous studies using the Minimal Model approach [32, 33] and strategies used to ameliorate this problem require either Bayesian techniques [34, 35] or arduous, clinically intense, frequently sampled protocols. However, it has been shown that fixing the glucose dependent clearance term (that has limited clinical value) maximises identification stability and allows the considerably less intense protocol of the DISST to produce a stable and relevant metric of insulin sensitivity $[4,12]$. The overall reduction in clinical intensity and improved parameter stability offered by the DISST comes at the cost of increased parameter identification complexity. However, this is a positive development, as it allows a lower per participant cost than the established, simple-model, intense-protocol tests for insulin sensitivity.

More intensive tests such as the glucose clamp [36] and the IVGTT [37] require specialist training for those performing the tests, involve a greater participant burden and are more costly, all of which generally limit their use to small research studies. They appear to be 
comparable tests, although the IVGTT, with a coefficient of variation of 14 to $30 \%$, is less reliable than the glucose clamp, coefficient of variation of 6 to 10\%. The particularly high repeatability has earned the glucose clamp gold standard status [16]. However, the glucose clamp yields different results at different infusion rates which complicates comparisons between studies [20,21]. The basic glucose clamp assumes all endogenous glucose and insulin secretion is fully suppressed, that all glucose uptake is mediated by insulin and that the uptake rate is proportional to the plasma insulin concentration [36]. In fact, insulin independent glucose uptake occurs and can be constant (to the brain and the central nervous system) or dependent on glucose concentration [38]. This is accounted for by the DISST [3].

An earlier study involving repeated tests demonstrated that the DISST was as reliable as the glucose clamp in measuring insulin sensitivity [2]. We report here a strong correlation between insulin sensitivity measured by the DISST and the glucose clamp $(\mathrm{R}=0.82)$. It is noteworthy that on average the DISST only under-estimated the clamp ISI by $0.1 \cdot 10^{-}$ ${ }^{2} \cdot \mathrm{mg} \cdot \mathrm{l} \cdot \mathrm{kg}^{-1} \cdot \mathrm{min}^{-1} \cdot \mathrm{pmol}^{-1}$, even though there were substantial differences between the two protocols. The ROC analysis, which is usually used to compare two very different tests, indicates that the DISST and the glucose clamp are reasonably comparable. Although both tests relate the rate of glucose uptake to an insulin concentration, the clamp involves a steady state, hyper-physiological protocol with suppression of insulin and glucose production and 23 hours of frequent sampling, whereas the DISST protocol involves only 35-minutes of less frequent sampling and does not significantly suppress endogenous insulin or glucose production. In contrast to the clamp, the DISST insulin sensitivity is a function of interstitial insulin and is measured with glucose and insulin concentrations that are typical of daily life. Furthermore, the DISST accounts for non-insulin mediated glucose uptake, which the clamp assumes is negligible. Thus, while the clamp was designed to maximise repeatability, the DISST was designed to be relevant to the participant's metabolic physiology.

In conclusion, we believe the DISST is a relatively low cost, practical test which yields substantially more information regarding glucose and insulin responses to stimuli than other available tests. DISST is safe and reliable and allows a reasonable estimation of insulin sensitivity. In addition estimates of insulin secretion can be obtained at the same time. It is a test which could be applied in clinical or research settings; either where a glucose clamp might be used or in larger trials where either an OGTT or the HOMA would be used. If the DISST were to be applied widely, it could greatly enhance our understanding of the 
332 pathophysiology of type 2 diabetes mellitus and help to more clearly differentiate the very

333 heterogeneous group who are at risk of type 2 diabetes mellitus.

334

335 Acknowledgements.

336 Dr Christopher Hann contributed to the development of the model-based parameter

337 identification process and the staff of the Endocrine Test Centre at Christchurch Hospital

338 provided clinical support for the study. An abstract based on this data has been published in

339 the American Diabetes Association Conference Proceedings 2010.

\section{Funding.}

342 This study was funded by the Health Research Council of New Zealand.

\section{Disclosure Statement.}

345 The authors declare that there is no conflict of interest associated with this manuscript.

\section{Author contributions.}

348 KAM contributed to study concept and design, to acquisition of data, analysis and 349 interpretation of data and the writing of the manuscript. JEB contributed to acquisition of 350 data and reviewed manuscript. PDD contributed to acquisition of data, analysis and 351 interpretation of data and contributed to writing of manuscript. TFL contributed to study 352 concept and design, pilot work which forms the basis of this paper and revised the 353 manuscript. LTM contributed to acquisition of data and contributed to writing of the manuscript. GMS contributed to study concept and design and acquisition of data and revised manuscript. SMW was the statistician and contributed to study design, analysis and interpretation of data and contributed to writing of the manuscript. JGC contributed to study concept and design, analysis and interpretation of data and contributed to the writing of the manuscript. JIM contributed to study concept and design, analysis and interpretation of data and contributed to the writing of the manuscript. 


$$
\begin{array}{ll}
\text { Plasma } & \quad \dot{C}=k_{2} Y-\left(k_{1}+k_{3}\right) C+\frac{\text { uen }(t)}{V p} \\
\text { C-peptide } & \\
\text { Interstitial } & \dot{Y}=k_{1} C-k_{2} Y \\
\text { C-peptide } &
\end{array}
$$

Plasma

Insulin

Interstitial

Insulin

$$
\dot{I}=\frac{n_{I}}{V p}(Q-I)-n_{K} I-n_{L} \frac{I}{1+\alpha_{I} I}+\frac{u e x(t)}{V p}+\left(1-x_{L}\right) \frac{u e n(t)}{V p}
$$

Glucose

$$
\dot{Q}=\frac{n_{I}}{V q} I-\left(\frac{n_{I}}{V q}+n_{C}\right) Q
$$

where: $k_{1-3}$ are kinetic parameters (1/min); $C$ and $Y$ are plasma and interstitial C-peptide concentrations, respectively (pmol/l); uen(t) is the time variant rate of insulin production (pmol/min); $I$ and $Q$ are the plasma and interstitial insulin concentrations respectively (pmol/l); $V p$ and $V q$ are the distribution volumes of insulin in the plasma and interstitium respectively (l); $n_{K}$ is the rate of insulin clearance by the kidney (1/min); $n_{I}$ is the transition rate of insulin between the plasma and interstitium (l/min); $n_{L}$ is the rate of hepatic insulin clearance $\left(\mathrm{min}^{-1}\right) ; \alpha_{I}$ is the saturation of hepatic insulin clearance $(1 / \mathrm{pmol}) ; n_{C}$ is the rate of insulin clearance to cells $(1 / \mathrm{min})$; $\operatorname{uex}(t)$ is the bolus input of insulin (pmol); $x_{L}$ is the hepatic first pass extraction of insulin (1); $p_{g u}$ is the glucose dependent (insulin-independent) rate of glucose disposal (1/min); SI is the modelled insulin sensitivity (l/pmol/min); $P$ is the glucose bolus (mmol); $\mathrm{Vg}$ is the volume of distribution of glucose (l); G is the glucose concentration

The measured C-peptide, insulin and glucose data is used to identify participant-specific parameters with methods that have been exhaustively defined and justified in previous 
384 publications $[2-4,10]$. However, the methods will be summarised in brief: Initially, a false 385 basal data point with concentrations equal to the measured basal sample was added 386 immediately prior to the glucose bolus. This ensured that the influence of the basal period on 387 the identified variables was equal across participants. The kinetic parameters of Equations 1 388 and 2 are quantified using functions of participant weight, height sex and age that were 389 defined by Van Cauter et al. [5]. A piece-wise linear interpolation of the C-peptide data was 390 used with these values in a deconvolution of Equations 1 and 2 to produce an endogenous 391 insulin production profile (uen $(t))$. Finally, $S I, V g, n_{L}$ and $x_{L}$ were identified using the 392 deconvoluted endogenous insulin production profile, insulin and glucose data, Equations 3-5 393 and the integral method $[3,10]$. Note that the $t=10$ minute glucose sample is assumed to be 394 affected by mixing and is thus ignored in the identification of $S I$ and $\mathrm{Vg}$ and is omitted from 395 Figure 4. 


\section{References}

3981 Lotz TF, Chase JG, McAuley KA, et al. Transient and steady-state euglycemic clamp 399 validation of a model for glycemic control and insulin sensitivity testing. Diabetes $400 \quad$ Technol Ther 2006;8(3):338-46

4012 Lotz TF, Chase JG, McAuley KA, et al. Monte Carlo analysis of a new model-based 402

403 method for insulin sensitivity testing. Comput Methods Programs Biomed 2008;89(3):215-25

404

3 Lotz TF, Chase JG, McAuley KA, et al. Design and clinical pilot testing of the model405 406 based dynamic insulin sensitivity and secretion test (DISST). J Diabetes Sci Technol 2010;4(6):1408-23

407

Lotz T. High resolution clinical model-based assessment of insulin sensitivity. (2007)

408

409 PhD Thesis Department of Mechanical Engineering, University of Canterbury, Christchurch, New Zealand, http://hdl.handle.net/10092/1571

Van Cauter E, Mestrez F, Sturis J, et al. Estimation of insulin secretion rates from Cpeptide levels. Comparison of individual and standard kinetic parameters for Cpeptide clearance. Diabetes 1992;41(3):368-77

413

ADA. Diagnosis and Classification of Diabetes Mellitus. Diabetes Care 2006;29(suppl_1):S43-S48

Matsuda M and DeFronzo RA. Insulin sensitivity indices obtained from oral glucose tolerance testing: comparison with the euglycemic insulin clamp. Diabetes Care 1999;22(9):1462-1470 Matthews DR, Hosker JP, Rudenski AS, et al. Homeostasis model assessment: insulin resistance and beta-cell function from fasting plasma glucose and insulin concentrations in man. Diabetologia 1985;28(7):412-9

4219 McAuley KA, Williams SM, Mann JI, et al. Diagnosing insulin resistance in the general population. Diabetes Care 2001;24(3):460-4 
42310 Hann CE, Chase JG, Lin J, et al. Integral-based parameter identification for long-term 424 dynamic verification of a glucose-insulin system model. Comput Methods Programs 425 Biomed 2005;77(3):259-70

42611 Lotz TF, Goltenbolt U, Chase JG, et al. A minimal C-peptide sampling method to 427 capture peak and total prehepatic insulin secretion in model-based experimental 428 insulin sensitivity studies. Journal of Diabetes Science and Technology 2009;3(4):875$429 \quad 86$

43012 Docherty PD, Chase JG, Lotz TF, et al. Evaluation of the performances and costs of a 431 spectrum of DIST protocols. Eds E. Carson UK International Conf on Control $432 \quad$ Coventry, UK 2010 6-pages

$433 \quad 13 \quad$ Ferrannini E and Mari A. How to measure insulin sensitivity. J Hypertens 434 1998;16(7):895-906

43514 Barrett EJ, Eggleston EM, Inyard AC, et al. The vascular actions of insulin control its 436 delivery to muscle and regulate the rate-limiting step in skeletal muscle insulin action. Diabetologia 2009;52(5):752-64

43815 Assmann G, Schriewer H, Schmitz G, et al. Qunatification of high-density-lipoprotein 439 cholesterol by precipitation with phosphotungstic acid/MgCl2. Clin Chem 1983;29(12):2026-30

16 Pacini G and Mari A. Methods for clinical assessment of insulin sensitivity and betacell function. Best Pract Res Clin Endocrinol Metab 2003;17(3):305-22

$443 \quad 17$ Ferrannini E. Insulin resistance is central to the burden of diabetes. Diabetes Metab $444 \quad$ Rev 1997;13(2):81-6

44518 Cobelli C, Toffolo G, Dalla Man C, et al. Assessment of beta-cell function in humans, 446 simultaneously with insulin sensitivity and hepatic extraction, from intravenous and oral glucose tests. Am J Physiol Endocrinol Metab 2007;293(1):E1-E15

44819 Docherty PD, Chase JG, Hann CE, et al. The identification of insulin saturation effects 449 during the dynamic insulin sensitivity test. Open Med. Inform. J. 2010;4:141-148 
45020 Natali A, Gastaldelli A, Camastra S, et al. Dose-response characteristics of insulin 451 action on glucose metabolism: a non-steady-state approach. Am J Physiol Endocrinol $452 \quad$ Metab 2000;278(5):E794-801

45321 Laakso M, Edelman SV, Brechel G, et al. Decreased effect of inuslin to stimulate 454 skeletal muscle blood flow in Obese man: A novel mechanism for insulin resistance. J. 455 Clin. Invest. 1990;85(6):1844-52

$45622 \quad$ Ferrannini E and Mari A. Beta cell function and its relation to insulin action in 457 humans: a critical appraisal. Diabetologia 2004;47(5):943-56

45823 Bock G, Dalla Man C, Campioni M, et al. Pathogenesis of pre-diabetes: mechanisms 459 of fasting and postprandial hyperglycemia in people with impaired fasting glucose $460 \quad$ and/or impaired glucose tolerance. Diabetes 2006;55(12):3536-49

46124 Jones CN, Pei D, Staris P, et al. Alterations in the glucose-stimulated insulin secretory 462 dose-response curve and in insulin clearance in nondiabetic insulin-resistant individuals. J Clin Endocrinol Metab 1997;82(6):1834-8

25 Mari A. Assessment of insulin sensitivity and secretion with the labelled intravenous 465 glucose tolerance test: improved modelling analysis. Diabetologia 1998;41(9):1029-39

26 Nyenwe EA, Jerkins TW, Umpierrez GE, et al. Management of type 2 diabetes: evolving strategies for the treatment of patients with type 2 diabetes. Metabolism 2011;60(1):1-23 naive Chinese type 2 diabetes mellitus. Metabolism 2010;59(6):780-786 Risk of Type 2 Diabetes from a Five-Year Prospective Study of 6784 Dansih People (Inter99). J diabetes Sci Technol. 2009;3(4):748-55 measurement of in vivo insulin-stimulated glucose disposal in humans: comparison of this approach with the insulin clamp and minimal model techniques. J Clin Endocrinol Metab 1985;60(4):723-6 
$47830 \quad$ Steil GM, Rebrin K, Hariri F, et al. Interstitial fluid glucose dynamics during insulin-

479 induced hypoglycaemia. Diabetologia 2005;48(9):1833-40

48031 Galvin P, Ward GM, Walters JM, et al. A simple method for quantitation of insulin 481 sensitivity and insulin release form an intravenous glucose tolerance test. Diabet Med 482 1992;9(10):921-8

$483 \quad 32$ Pillonetto G, Sparacino G, Magni P, et al. Minimal model S(I)=0 problem in NIDDM 484 subjects: nonzero Bayesian estimates with credible confidence intervals. Am J Physiol 485 Endocrinol Metab 2002;282(3):E564-573

48633 Caumo A, Vicini P, Zachwieja JJ, et al. Undermodeling affects minimal model 487 indexes: insights from a two-compartment model. Am J Physiol 1999;276(6 Pt 488 1):E1171-1193

48934 Callegari T, Caumo A and Cobelli C. Bayesian two-compartment and classic single490 compartment minimal models: comparison on insulin modified IVGTT and effect of 491 experiment reduction. IEEE Trans Biomed Eng 2003;50(12):1301-9

49235 Erichsen L, Agbaje OF, Luzio SD, et al. Population and individual minimal modeling 493 of the frequently sampled insulin-modified intravenous glucose tolerance test. 494 Metabolism 2004;53(10):1349-54

49536 DeFronzo RA, Tobin JD and Andres R. Glucose clamp technique: a method for 496 quantifying insulin secretion and resistance. Am J Physiol 1979;237(3):E214-23

49737 Bergman RN, Ider YZ, Bowden CR, et al. Quantitative estimation of insulin 498 sensitivity. Am J Physiol 1979;236(6):E667-77

49938 Zierler K. Whole body glucose metabolism. Am J Physiol 1999;276(3 Pt 1):E409-426 500 
Table 1. Correlation between the DISST insulin sensitivity and variables known to be associated with insulin resistance as well as two simple surrogates for assessing insulin sensitivity, the HOMA and the Matsuda OGTT.

504

\begin{tabular}{|l|l|l|l|l|}
\hline & $\begin{array}{l}\text { Mean } \\
(\mathrm{n}=123)\end{array}$ & SD & $\begin{array}{l}\text { Correlation with } \\
\text { the DISST SI }\end{array}$ & P value \\
\hline Age (years) & 42 & 12.2 & -0.16 & 0.09 \\
\hline Waist circumference (cm) & 95.5 & 14.9 & -0.51 & $<0.001$ \\
\hline BMI (kg/m $\left.{ }^{2}\right)$ & 31.7 & 6.90 & -0.45 & $<0.001$ \\
\hline FPG (mg/dl) & 86.4 & 8.64 & -0.34 & 0.002 \\
\hline Fasting triglycerides (mmol/l) & 1.30 & 0.94 & -0.27 & 0.002 \\
\hline Fasting HDL cholesterol (mmol/l) & 1.19 & 0.30 & 0.40 & $<0.001$ \\
\hline Fasting insulin (pmol/l) & 78.9 & 75.4 & -0.63 & $<0.001$ \\
\hline HOMA & 2.4 & 2.27 & -0.40 & $<0.001$ \\
\hline Matsuda OGTT & 16.9 & 11.0 & 0.56 & $<0.001$ \\
\hline
\end{tabular}




\begin{tabular}{|c|c|c|c|c|}
\hline & $\begin{array}{l}0-4250 \\
\text { pmol of } \\
\text { insulin } \\
(n=41)\end{array}$ & $\begin{array}{l}4251-7000 \\
\text { pmol of } \\
\text { insulin } \\
(n=42)\end{array}$ & $\begin{array}{l}\text { 7001- } 22000 \\
\text { pmol of } \\
\text { insulin } \\
(n=40)\end{array}$ & $\begin{array}{l}\mathrm{P} \\
\text { value }\end{array}$ \\
\hline Age (years) & $44(12)$ & $42(12)$ & $38(13)$ & 0.09 \\
\hline Gender \% Female & 82 & 74 & 83 & 0.51 \\
\hline Weight (kg) & $84.5(18.3)$ & $86.2(18.0)$ & $93.4(24.6)$ & 0.12 \\
\hline Waist (cm) & $93.0(15.0)$ & $93.6(12.7)$ & $100.1(16.1)$ & 0.06 \\
\hline BMI $\left(\mathrm{kg} / \mathrm{m}^{2}\right)$ & $30.4(6.6)$ & $30.9(6.0)$ & $33.7(7.7)$ & 0.06 \\
\hline $\mathrm{SBP}(\mathrm{mmHg})$ & $120(14)$ & $120(14)$ & $123(19)$ & 0.69 \\
\hline DBP (mmHg) & $76(10)$ & $77(11)$ & $77(8)$ & 0.89 \\
\hline Fasting triglycerides (mmol/l) & $1.30(1.40)$ & $1.21(0.57)$ & $1.39(0.64)$ & 0.68 \\
\hline Fasting HDL cholesterol (mmol/l) & $1.24(0.29)$ & $1.22(0.30)$ & $1.11(0.29)$ & 0.09 \\
\hline FPG (mg/dl) & $86.4(9.0)$ & $82.8(7.2)$ & $82.8(7.2)$ & 0.01 \\
\hline Fasting insulin (pmol/L) & $61.6(38.7)$ & $66.9(40.9)$ & $109.1(114.5)$ & 0.007 \\
\hline IGT \% & 7 & 12 & 15 & 0.54 \\
\hline Insulin sensitivity (DISST) $^{\mathrm{a}}$ & $1.2(0.69)$ & $1.3(0.69)$ & $0.9(0.48)$ & 0.06 \\
\hline
\end{tabular}

Table 2. Clinical and biochemical measures by tertiles of first phase of insulin release (AUC $_{10}$, from 5 to 15 minutes) during the DISST ( $\left.\mathrm{n}=123\right)$. 
512 Table 3. Clinical and biochemical measures by tertiles of second phase of insulin release

$513 \quad\left(\mathrm{AUC}_{2 \mathrm{nd}}\right.$, from 15 to 35 minutes) during the DISST ( $\left.\mathrm{n}=123\right)$.

\begin{tabular}{|l|l|l|l|l|}
\hline & $\begin{array}{l}0-5000 \\
\text { pmol of insulin } \\
(\mathrm{n}=44)\end{array}$ & $\begin{array}{l}5001-8000 \\
\text { pmol of insulin } \\
(\mathrm{n}=38)\end{array}$ & $\begin{array}{l}8001-16000 \\
\text { pmol of insulin } \\
(\mathrm{n}=41)\end{array}$ & P value \\
\hline Age (years) & $40(12.8)$ & $40(11.4)$ & $45(12.0)$ & 0.12 \\
\hline Gender \% Female & 80 & 79 & 80 & 0.98 \\
\hline Weight (kg) & $76.6(12.1)$ & $86.0(14.0)$ & $102.2(24.8)$ & $<0.001$ \\
\hline Waist (cm) & $84.9(8.5)$ & $94.8(11.5)$ & $107.4(14.3)$ & $<0.001$ \\
\hline BMI (kg/m $\left.{ }^{2}\right)$ & $27.2(4.2)$ & $31.3(4.7)$ & $36.8(7.5)$ & $<0.001$ \\
\hline SBP (mmHg) & $118(14)$ & $119(14)$ & $126(18)$ & 0.04 \\
\hline DBP (mmHg) & $74(9)$ & $76(8)$ & $79(12)$ & 0.05 \\
\hline $\begin{array}{l}\text { Fasting triglycerides } \\
\text { (mmol/l) }\end{array}$ & $0.86(0.29)$ & $1.57(1.40)$ & $1.51(1.51)$ & 0.005 \\
\hline $\begin{array}{l}\text { Fasting HDL cholesterol } \\
\text { (mmol/l) }\end{array}$ & $1.29(0.26)$ & $1.29(0.32)$ & $0.99(0.19)$ & $<0.001$ \\
\hline FPG (mg/dl) & & & & \\
\hline Insulin (pmol/l) & $79.2(7.2)$ & $82.8(7.2)$ & $88.2(7.2)$ & $<0.001$ \\
\hline IGT \% & $40.0(19.2)$ & $67.1(24.3)$ & $131.5(108.5)$ & $<0.001$ \\
\hline Insulin sensitivity (DISST) & $1.6(0.69)$ & $1.1(0.39)$ & $0.7(0.25)$ & $<0.001$ \\
\hline Measur) & 13 & & \\
\hline
\end{tabular}

${ }^{\mathrm{a}}$ Measured in $10^{-4} \cdot \mathrm{l} \cdot \mathrm{pmol}^{-1} \cdot \mathrm{min}^{-1}$. 
516 Table 4. Measures indicating $\beta$ cell function by glucose tolerance status ( $n=123)$.

\begin{tabular}{|l|l|l|l|l|}
\hline & $\mathrm{n}=$ & $\begin{array}{l}\text { Basal Insulin } \\
\text { Production } \\
\left(U_{b}\right)^{\mathrm{a}} \\
(\mathrm{pmol} / \mathrm{min}) . \\
\text { Mean (SD) }\end{array}$ & $\begin{array}{l}\text { First Phase } \\
\text { Insulin Secretion } \\
\left(\mathrm{AUC}_{10}\right)^{\mathrm{b}} \\
(\mathrm{pmol}) . \\
\text { Mean (SD) }\end{array}$ & $\begin{array}{l}\text { Second Phase } \\
\text { Insulin Secretion } \\
\left(\mathrm{AUC}_{2 \mathrm{nd}}\right)^{\mathrm{c}} \\
(\mathrm{pmol}) . \\
\text { Mean (SD) }\end{array}$ \\
\hline All data & 123 & $235(103)$ & $6,060(3,564)$ & $6,889(3,320)$ \\
\hline NGT & 109 & $230(105)$ & $5,973(3,578)$ & $6,660(3,245)$ \\
\hline IGT & 14 & $276(74)$ & $6,739(3,502)$ & $8,668(3,487)$ \\
\hline P value & & 0.11 & 0.45 & 0.03 \\
\hline
\end{tabular}

$517{ }^{\mathrm{a}} U_{b}$ is the basal rate of insulin production; ${ }^{\mathrm{b}} \mathrm{AUC}_{10}$ is the amount of insulin produced 10

518 minutes after the glucose bolus above the basal rate; ${ }^{\mathrm{c}} \mathrm{AUC} \mathrm{Cnd}_{2 \mathrm{n}}$ is the total amount of insulin 519 produced between $t=15$ and 35 minutes. 


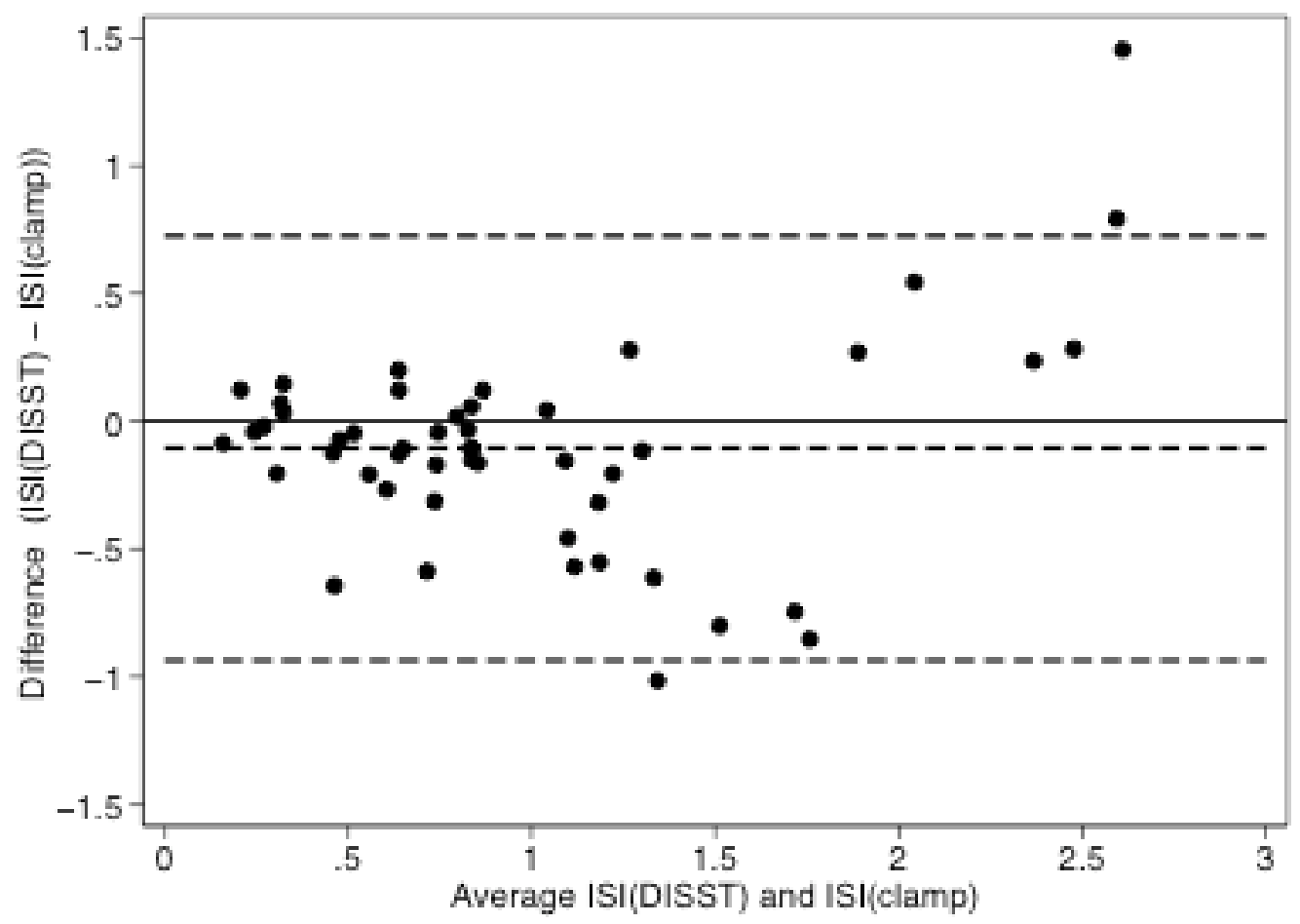

521

522 Figure 1.

523 
524

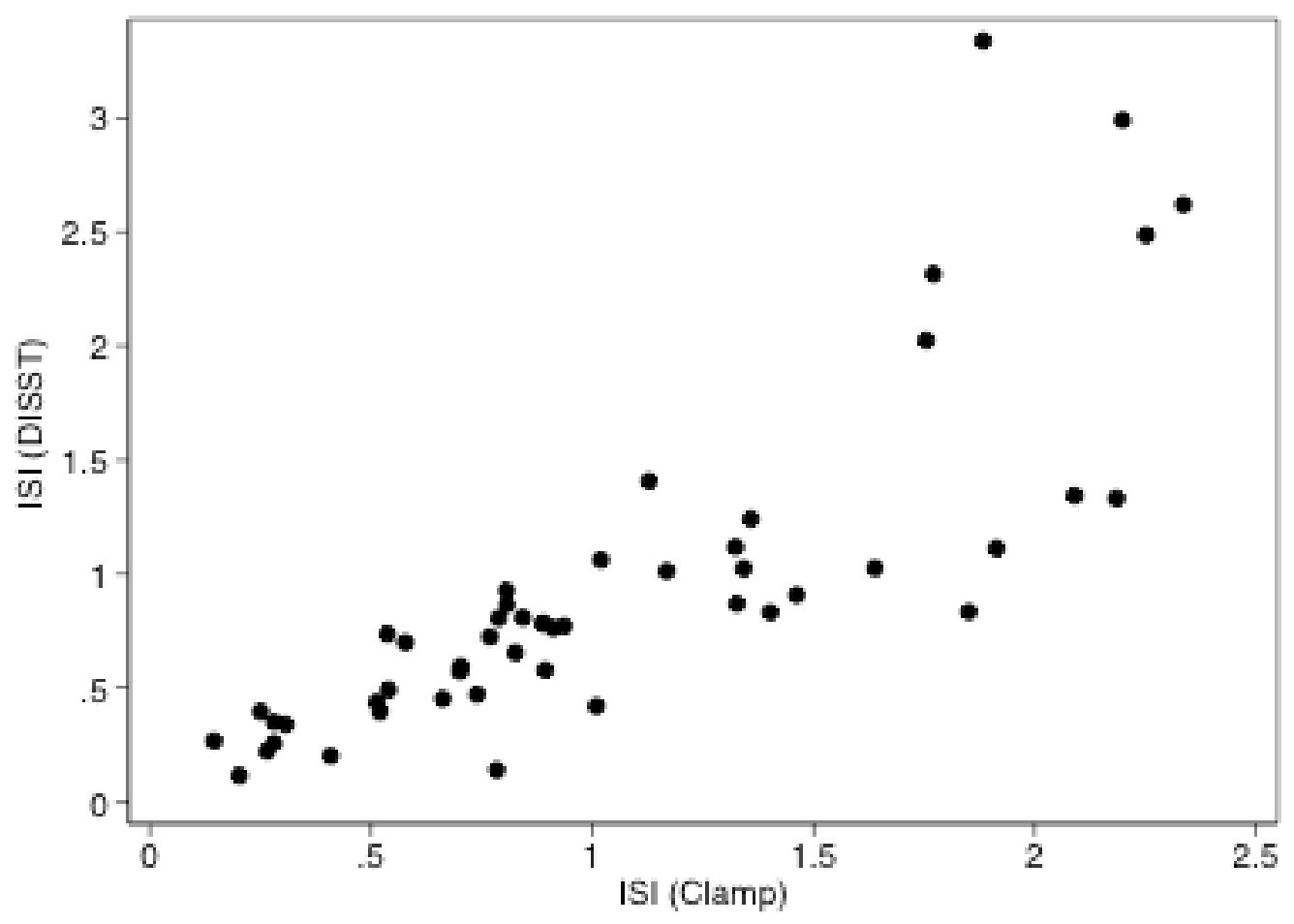

525

$526 \quad$ Figure 2.

527 


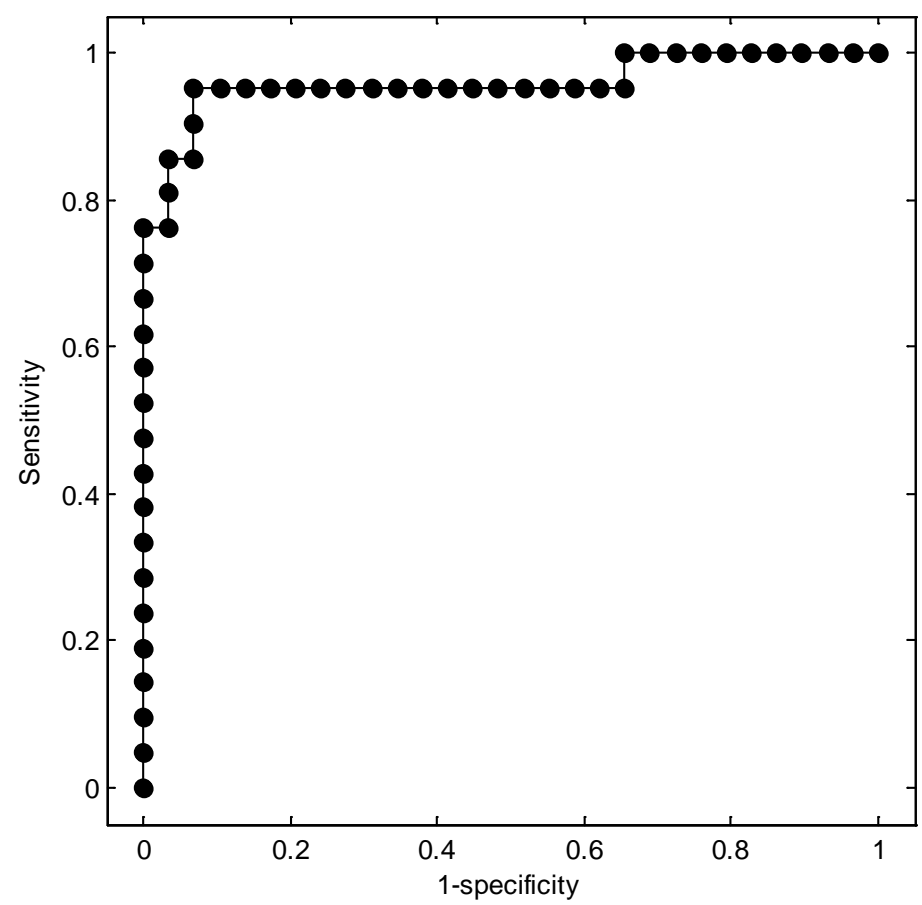

528

$529 \quad$ Figure 3.

530 

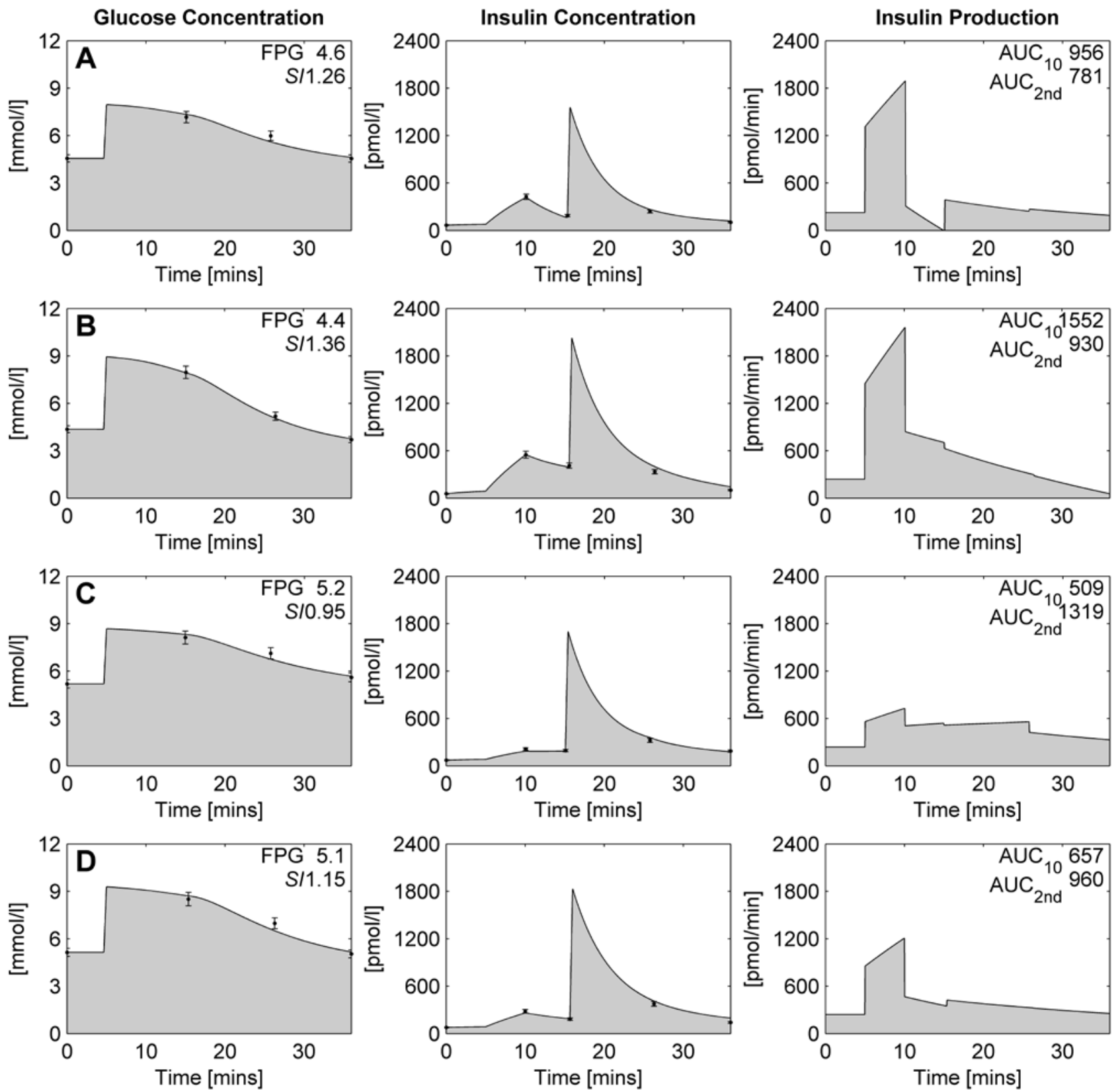

\section{$532 \quad$ Figure 4.}




\section{Figure Legends}

535

536 Figure 1. The Bland Altman plot of insulin sensitivity estimates derived from the DISST and 537 the glucose clamp, showing the bias between the two tests, with the DISST overestimating the 538 glucose clamp insulin sensitivity estimate by $0.1 \cdot 10^{-2} \cdot \mathrm{mg} \cdot 1 \cdot \mathrm{kg}^{-1} \cdot \mathrm{min}^{-1} \cdot \mathrm{pmol}^{-1}(95 \% \mathrm{CI}-0.2$ to 5390.0 ). The limits of agreement are -0.9 to $0.7 \cdot 10^{-2} \cdot \mathrm{mg} \cdot 1 \cdot \mathrm{kg}^{-1} \cdot \mathrm{min}^{-1} \cdot \mathrm{pmol}^{-1}$. 540

541 Figure 2. The correlation of the DISST and the glucose clamp insulin sensitivity values (units 542 are $\left.10^{-2} \cdot \mathrm{mg} \cdot \mathrm{l} \cdot \mathrm{kg}^{-1} \cdot \mathrm{min}^{-1} \cdot \mathrm{pmol}^{-1}\right)$.

543

544 Figure 3. ROC curve of the DISST against the gold standard, the glucose clamp, c index $545=0.96$.

546

547 Figure 4. Blood glucose, plasma insulin and insulin production responses of four individuals 548 to the DISST stimulus. The second peak of the insulin concentration is due to the exogenous 549 bolus of insulin used in the DISST protocol.

550 